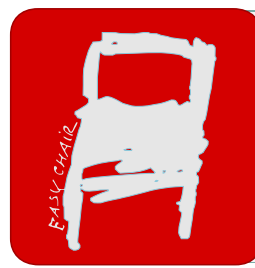

EPiC Series in Health Sciences

Volume 2, 2018, Pages 183-186

CAOS 2018. The 18th Annual Meeting of the International Society for Computer Assisted Orthopaedic Surgery

\title{
An augmented reality app for lower limb exercise- A focus group study
}

\author{
Shameem AC Sampath ${ }^{1,}$, Howard $\mathrm{Ho}^{3}$ and Min-Liang ${ }^{2}$ \\ ${ }^{1}$ Bluespot The Knee Clinic, Lytham, UK \\ ${ }^{2}$ Medical of Science and Technology, Taipei, Taiwan \\ ${ }^{3}$ Medical of Science and Technology, Seattle, USA \\ bluespotkneeclinic@me.com, apphoward10@gmail.com, \\ pml.wang@gmail.com
}

\begin{abstract}
Although exercises improve the outcome of patients with osteoarthritis, patient compliance is poor. Behaviour modification and measuring compliance remain the Holy Grail of healthcare professionals. The responses of a focus group of 20 volunteers to a novel automatic compliance recording augmented reality exercise app were studied. Full compliance was achieved in all cases and was measured and recorded by the app. Overall, the app itself received favorable responses regarding the user interface and user experience.
\end{abstract}

\section{Introduction}

Physiotherapy and exercise are accepted as being beneficial for both prehab and postoperative/post injury rehabilitation. The benefits include pain reduction and delaying or removing the need for surgery (Fransen et al, 2015). Studies show that up to $70 \%$ of patients do not do their exercises as prescribed, (Szöts, K. 2015). The reasons include a lack of understanding or forgetting to how or how often to do them, pressures of work or social life and simply finding them boring. Behaviour modification and measuring compliance remain the Holy Grail of healthcare professionals.

Increasing demands on reducing healthcare resources have made this a priority for governments across the globe. There is, however, a gap in the literature showing automatic measurement of compliance by stand-alone mobile apps.

Gamification has been seen as an adjunct to behaviour modification and a means of sustaining the interest of the target patient population (Edney et al, 2017). The widespread availability and increasing processing power of smartphones have facilitated the development of augmented reality 
apps. The distribution of smartphone processing power, however, tends to correlate with the underlying operating system, age, income and social demographics.

Therefore, for an app to maximise its reach, it must have the ability to function effectively across this current wide spectrum of available technologies and demographic groups. Above all, the app should be easy to use. The purpose of this study was to made an initial determination of patient acceptability and the opportunity for behaviour modification with regard to using Quads AR for lower limb exercises.

Quads AR is an augmented reality app for lower limb exercise. It encourages the user to perform variations of lower limb movements through gamification (Figure 1). The games were designed to encourage slow steady muscle contractions (holding mode) and fast twitch muscle contractions (hitting mode) to activate the Type I and Type II muscles fibres respectively Moreover, the app automatically measures and records compliance. The user has the option to share the data and screenshots by email to health care professionals or on social media.

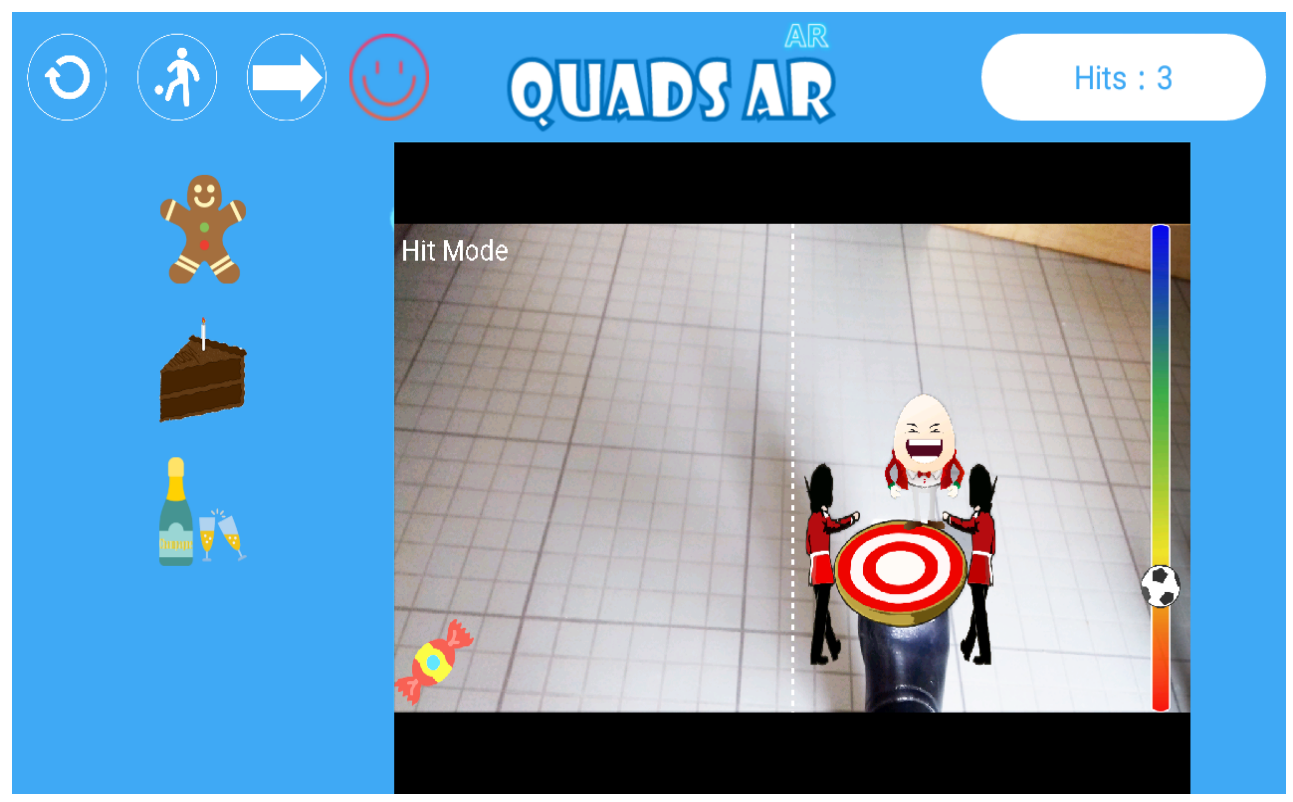

Figure 1. Screen shot showing the interaction of the user's foot with virtual objects and reward system.

The core functionality is the ability to interact with a variety of augmented reality objects using the interaction of the user's foot with virtual objects superimposed on a live real world visual field. There are two options for foot tracking. The markerless option uses computer vision and is suitable for higher end smartphones with faster processors. The marker option uses a marker stuck on the user's sock or shoe. It is suitable for the still larger number of older smartphones with slower processors. 


\section{Methods}

This initial study comprised a focus group of 20 volunteers. They were given the app using the marker to try on a smartphone after basic instructions. Compliance was automatically recorded by the app. They were then each given a paper questionnaire with 10 closed format rating scale questions and one free text comment section to record their responses. The responses were graded from 1 to 5 corresponding to strongly disagree, disagree, neutral, agree, strongly agree respectively.

\section{Results}

There were 10 males and 10 females. The ages ranged from 16 to 78 years, mean age 58.8 years (std dev 21.4). 4 volunteers did not give their ages. There was $100 \%$ compliance as recorded by the app. The responses to each question are given in Table 1.

\begin{tabular}{|l|c|}
\hline & Mean \\
\hline 1. The instructions are easy to understand & 4.2 \\
\hline 2. I was able to learn to play the game reasonably easily & 4.8 \\
\hline 3. The game is fun and interesting & 4.2 \\
\hline 4. I was able to attach and remove the sticker easily to my shoe & 3.9 \\
\hline 5. The game can make doing exercises fun & 4.2 \\
\hline 6. The game may encourage people to do their exercises & 4.3 \\
\hline 7. I would like to see more games like this & 4.5 \\
\hline $\begin{array}{l}\text { 8. I would like to see this available on the National Health } \\
\text { Service }\end{array}$ & 4.4 \\
\hline 9. I could feel the effect on my thigh muscles after playing the & 4.4 \\
\hline $\begin{array}{l}\text { 10. I think this game may help strengthen my muscles if played } \\
\text { regularly }\end{array}$ & 4.6 \\
\hline Strongly disagree 1, disagree 2, neutral 3, agree 4, strongly agree 5 & \\
\hline
\end{tabular}

Table 1. A summary of the results of the focus group questionnaire on the usability of Quads AR

Free Text: There were only three responses in this section:

1. "maybe the type of game needs to be related to the age group"

2. "excellent- Fantastic simple idea, very cost effective, fun to do, distracts from pain" 


\section{3. "a good encouraging method"}

\section{Conclusion}

Overall the responses were informative and favourable towards the use of the augmented reality app. This is consistent with other studies of gamefied exercises (Bardus et al, 2016).

It is significant that $6 / 20$ users did not agree that the marker stickers were easy to apply and remove. This finding led to the development of the markerless computer vision version. Both versions are now currently available on the android and iOS platforms. The first comment also been heeded and as a result further games aimed at different age groups are being developed. Again, this is consistent with previous work (Miller et al, 2016). The favourable disposition towards the use of the app was supported by the data recorded by the app itself which showed compliance in all cases.

Although this small initial study has provided valuable information, suitably large, and randomised control trials are need. Further studies looking at accuracy, precision, efficiency and the EMG responses of individual muscle groups are planned. Random controlled studies with preOp and postOp patients and controls are planned

\section{References}

Bardus M, van Beurden SB, Smith JR, Abraham C. (2016;13:1-9). A review and content analysis of engagement, functionality, aesthetics, information quality, and change techniques in the most popular commercial apps for weight management. Int J Behav Nutr Phys Act

Edney S, Plotnikoff R, Vandelanotte C, Olds T, De Bourdeaudhuij I, Ryan J, Maher C. (2017 Nov 2;17(1):859. doi: 10.1186/s12889-017-4882-7) "Active Team" a social and gamified app-based physical activity intervention: randomised controlled trial study protocol. BMC Public Health. 2017 Nov 2;17(1):859. doi: 10.1186/s12889-017-4882-7.

Fransen M, McConnell S, Harmer AR, Van der Esch M, Simic M, Bennell KL (2015 Jan 9;1:CD004376. doi: 10.1002/14651858.CD004376.pub3). Exercise for osteoarthritis of the knee. Cochrane Database Syst Rev.

Miller AS, Cafazzo JA, Seto E. (2016;22:184-93). A game plan: gamification design principles in mhealth applications for chronic disease management. Health Inform J.

Szöts, K. (2015, 19, p36). Physical health problems experienced in the early postoperative recovery period following total knee replacement. Int. J. Orthopaedic and Trauma Nursing 